\title{
Deux langues officielles !
}

Une communauté bilingue. Le cas de la Catalogne

Joan Pere et Marçal Fité

\section{OpenEdition}

\section{Journals}

Édition électronique

URL : http://journals.openedition.org/ries/2866

DOI : $10.4000 /$ ries. 2866

ISSN : 2261-4265

Éditeur

Centre international d'études pédagogiques

Édition imprimée

Date de publication : 1 septembre 1998

Pagination : 53-60

ISSN : $1254-4590$

Référence électronique

Joan Pere et Marçal Fité, « Deux langues officielles! », Revue internationale d'éducation de Sèvres [En ligne], 19 | septembre 1998, mis en ligne le 18 avril 2013, consulté le 23 mars 2021. URL : http:// journals.openedition.org/ries/2866; DOI : https://doi.org/10.4000/ries.2866

Ce document a été généré automatiquement le 23 mars 2021

(C) Tous droits réservés 


\section{Deux langues officielles!}

Une communauté bilingue. Le cas de la Catalogne

Joan Pere et Marçal Fité

\section{Les langues dans la société et à l'école}

1 La Catalogne est l'une des Communautés autonomes de l'État espagnol qui possède sa propre langue, langue reconnue scientifiquement et juridiquement: le catalan. Le concept de langue propre trouve son origine dans le statut d'autonomie de la Catalogne (1979) et fait référence à l'enracinement historique de la langue catalane sur le territoire. C'est pourquoi cette communauté possède deux langues officielles: le catalan, langue maternelle d'un peu plus de la moitié de la population, et le castillan ${ }^{1}$.

Le castillan ou espagnol qui, selon la constitution de 1978, est la langue relationnelle de l'État espagnol, et donc la langue officielle, se trouve en contact étroit avec le catalan du fait même qu'il est aussi la langue maternelle d'une partie importante de la population de la Catalogne (près de $49 \%$ ), principalement les Catalans issus des territoires «castillanophones" du reste de l'Espagne de la première ou deuxième génération.

Dans le système éducatif, cette situation de contact et de "co-officialité » entre les langues catalane et castillane est réglementée par un cadre de lois qui établit le caractère préférentiel du catalan comme langue véhiculaire et d'apprentissage dans les étapes préscolaire, primaire et secondaire; il garantit aussi l'apprentissage des deux langues officielles: «la présence du catalan et du castillan doit être garantie dans les programmes, de telle manière que tous les enfants, quelle que soit leur langue habituelle au début de leur scolarité, aient la possibilité d'utiliser normalement et correctement les deux langues officielles à la fin de la scolarité obligatoire " (loi de Politique linguistique, 1997, art. 21.3). La loi établit aussi que, pour parvenir à ces objectifs, "les élèves ne devront pas être séparés dans les établissements ou les groupes-classes en fonction de leur langue habituelle» (art. 21.5) Ce cadre envisage aussi l'apprentissage obligatoire d'une langue étrangère à partir du cycle moyen de l'enseignement primaire, ainsi que la possibilité d'accéder à une seconde langue 
étrangère optionnelle à partir du cycle supérieur de l'enseignement primaire, dans le secondaire obligatoire et le bachillerato.

Structure du système éducatif espagnol

\author{
Enseignement préscolaire (jusqu'à 6 ans) \\ $1^{\text {er }}$ cycle ou " jardin d'enfants " \\ $2^{\mathrm{e}}$ cycle ou " maternelle » \\ Enseignement primaire (6 à 12 ans) \\ cycle initial (6 à 8 ans) \\ cycle moyen (8 à 10 ans) \\ cycle supérieur (10 à 12 ans) \\ Enseignement secondaire (12 à 18 ans) \\ enseignement secondaire obligatoire (ESO) (12 à 16 ans) \\ $1^{\text {er }}$ cycle (12 à 14 ans) \\ $2^{\mathrm{e}}$ cycle (14 à 16 ans) \\ enseignement secondaire post-obligatoire (16 à 18 ans) \\ bachillerato (16 à 18 ans) \\ formation technico-professionnelle de degré moyen (16 à 18 ans)
}

4 Les remarques précédentes permettent de comprendre que, dans une communauté bilingue comme la Catalogne, l'enseignement de la langue requiert une planification adéquate et une prise en compte de certains aspects étroitement liés à la situation sociolinguistique du territoire et au caractère de complémentarité et d'opposition existant entre les langues catalane et castillane.

C'est pourquoi on a prévu dans l'agencement des programmes, lorsqu'ont été fixés les contenus et les objectifs de l'enseignement de la langue, un certain degré de souplesse et d'autonomie pour que chaque établissement puisse tenir compte de la réalité sociolinguistique spécifique de ses élèves. Cela est rendu possible grâce au projet linguistique d'établissement dont les fonctions principales (toujours en accord avec le règlement établi) sont de :

- définir la fonction et les utilisations des deux langues officielles ;

- fixer les options pour les langues étrangères ;

- décider des critères généraux se rapportant au début de l'apprentissage de chacune des langues et à son développement tout au long du cursus.

La réalité de l'enseignement de la langue varie donc principalement en fonction des caractéristiques linguistiques des élèves et de l'environnement social des écoles; en effet, il faut savoir que les proportions d'élèves «catalano-phones" et " castillanophones » peuvent être très différentes selon les établissements et les zones géographiques (elles oscillent, par exemple, entre une nette prédominance du catalan dans les régions de Gérone ou de Lérida et une présence majoritaire du castillan dans les villes de la périphérie de Barcelone).

Dans les contextes majoritairement "catalanophones", on part généralement de la langue parlée à la maison et l'on commence donc l'enseignement préscolaire exclusivement en catalan; c'est toujours dans cette langue que se déroule l'apprentissage de la lecture et de l'écriture ainsi que le travail sur les aspects 
communicatifs et fonctionnels aussi bien que sur les aspects formels de la langue. La langue castillane est introduite dans le cycle initial de l'enseignement primaire et le travail didactique consiste essentiellement à garantir son utilisation formelle correcte, principalement par le biais de stratégies destinées à améliorer la fluidité, la phonétique, à élargir le lexique et à maîtriser la morphosyntaxe.

Dans les contextes "castillanophones", ce sont les Programmes d'immersion linguistique (PIL) qui, jusqu'à maintenant, ont donné les meilleurs résultats - surtout dans l'enseignement primaire - pour arriver à une bonne maîtrise des deux langues. L'implantation de ces programmes provient du fait que les possibilités d'utilisation extra-scolaire de la langue catalane dans des contextes « castillanophones » sont assez réduites, étant donné la domination presqu'absolue qu'y exerce la langue castillane, aussi bien dans la société que dans les moyens de communication. D'un point de vue pédagogique, les PIL sont caractérisés par la scolarisation en langue catalane, toujours accompagnée d'une prise en compte de la langue familiale de l'élève et de l'enseignement systématique de la langue castillane, dès le cycle initial. Ils partent de l'hypothèse $d^{\prime}$ " interdépendance linguistique ${ }^{2}$ " et sont basés sur un principe qui suppose que les deux langues ont une compétence sous-jacente commune ou interdépendante, si bien que l'expérience dans l'une des deux peut stimuler et développer la compétence sous-jacente de chacune d'entre elles.

Ces programmes sont suivis de façon assez généralisée tout au long de l'enseignement primaire, mais ils sont généralement modifiés dans le secondaire en accord avec les projets linguistiques de chaque établissement. Les PIL, il est vrai, ont été à certains moments l'objet de controverse politique ; pourtant, les résultats obtenus semblent les avaliser puisque les élèves qui les ont suivis améliorent non seulement leurs connaissances en langue catalane, mais aussi leur niveau d'acquisition de la langue castillane qui apparaît équivalent à celui des élèves « castillanophones » scolarisés en castillan, ainsi que l'ont souligné différentes études³.

Pour conclure sur ces points, nous dirons que la politique éducative de la Catalogne en ce qui concerne les langues part du principe que, dans les communautés bilingues, l'enseignement de la langue (ou plutôt l'enseignement linguistique) doit contribuer à l'extension de l'utilisation de la langue propre à chaque communauté (et, par conséquent, à la normalisation linguistique) non seulement par l'étude de la langue minoritaire mais aussi par l'apprentissage scolaire dans cette langue. Car une langue n'est pas seulement un outil de communication dans les usages linguistiques courants, elle est aussi un instrument essentiel dans l'acquisition des apprentissages, dans le développement de la pensée et dans la construction de l'identité socioculturelle des personnes et des peuples. C'est pourquoi il convient d'utiliser la langue minoritaire comme véhicule d'apprentissage scolaire et l'on comprend l'élaboration des différents Programmes d'immersion linguistique, dont la finalité est de favoriser l'acquisition de compétences linguistiques, discursives, sociolinguistiques et stratégiques dans la langue minoritaire. Puisqu'en fin de compte, l'objectif essentiel de l'enseignement linguistique dans les communautés bilingues n'est autre que de contribuer à l'acquisition du meilleur niveau possible de compétences communicatives dans les deux langues. 


\section{Le domaine de la langue dans l'enseignement secondaire}

11 L'enseignement des langues catalane et castillane et de la littérature aux différents niveaux éducatifs est conçu comme un seul domaine, obligatoire pour tous les élèves et ayant des objectifs communs et des contenus qui se recoupent. Dans l'étape de l'enseignement secondaire obligatoire, quinze crédits (450 heures de cours) obligatoires y sont consacrés, plus un certain nombre de crédits dits variables, compris généralement entre deux et cinq (60 à 150 heures). Dans le bachillerato (étape de 16 à 18 ans conduisant à l'obtention du diplôme final de l'enseignement secondaire), il y a six crédits (180 heures), auxquels il faut ajouter six crédits spécifiques de littérature pour certaines sections ainsi que quelques crédits variables (entre deux et quatre généralement). Dans tous les cas, il s'agit d'une enveloppe horaire globale que chaque établissement peut distribuer de façon spécifique tout au long du cursus.

\section{Enseignement secondaire obligatoire}

12 Dans ce cycle (12-16 ans), l'objectif fondamental du champ disciplinaire langue catalane/langue castillane/littérature est d'obtenir qu'à la fin de cette étape les élèves soient capables de comprendre et de produire des messages oraux et écrits de façon appropriée, autonome et créative dans les deux langues officielles, en les utilisant pour communiquer entre eux et pour organiser la pensée, et de réfléchir sur les processus qui entrent en jeu dans l'utilisation du langage. Les contenus que l'on donne à ce champ disciplinaire sont donc orientés vers la communication, centrés sur le développement d'une série de savoir-faire linguistiques en rapport avec la compréhension orale, la compréhension écrite, l'expression de messages oraux et la rédaction de textes écrits. On part du principe que le développement de ces savoir-faire aide les élèves à concrétiser leur pensée, à exprimer leurs sentiments, à pouvoir améliorer leur capacité relationnelle et à intervenir plus facilement dans la vie sociale. C'est le caractère communicatif et fonctionnel du programme qui est souligné lorsqu'il est catégoriquement affirmé que : «L'étude de ce champ disciplinaire doit être envisagée comme un tout qui ne peut avoir de sens que dans la compréhension et l'expression orales et écrites. Tel est le cadre général de référence dans lequel doivent être réinterprétées les différentes unités du travail scolaire de la langue ${ }^{4}$ ».

13 Les contenus de ce champ disciplinaire sont regroupés en six domaines : langue orale, langue écrite, littérature, lexique, grammaire, langue et société. Cependant, il est indiqué que les trois premiers domaines (langue orale, langue écrite et littérature) doivent représenter la plus grande partie de l'activité de cette discipline; leurs contenus forment donc la « colonne vertébrale » de tout le travail du cours de langue et littérature. Les autres domaines, par contre, apportent plutôt des contenus d'« appui » et leur place ne devrait pas être centrale ni prépondérante durant cette étape scolaire

Ce regroupement démontre clairement l'intégration de l'enseignement littéraire dans la perspective générale de l'apprentissage de la langue et de la communication, puisque le fait littéraire est considéré comme un fait de communication et que le discours littéraire est conçu comme un usage fonctionnellement distinct et non comme une langue différente de la langue commune ni comme l'utilisation la plus élaborée de la langue. Dans ce contexte, la littérature n'est plus conçue comme un répertoire de textes 
consacrés par la tradition académique et susceptibles d'être étudiés autour de l'axe diachronique de l'histoire ou d'être analysés avec des méthodologies complexes pour en percer les sens les plus cachés, mais comme un outil de communication grâce auquel les individus tentent de donner un sens à leur propre existence, d'éclaircir leur identité individuelle et collective et d'utiliser le langage de façon créative. L'enseignement littéraire consistera donc fondamentalement à choisir les stratégies les mieux adaptées pour stimuler l'acquisition d'habitudes de lecture chez les adolescents, pour favoriser un accès fluide aux textes, pour améliorer leur compréhension écrite et pour rendre possible de cette manière la construction de leur compétence culturelle au sein de nos sociétés.

Quant à l'intégration de la langue catalane et de la langue castillane dans un même champ disciplinaire, les orientations contenues dans le programme de cette étape éducative montrent que pour organiser en modules (par discipline) les contenus de toute cette étape, les professeurs devront planifier ensemble et coordonner leurs actions afin de rentabiliser les apprentissages acquis dans l'une ou l'autre langue. Il en est de même pour l'adaptation des programmes aux connaissances et aux compétences linguistiques des élèves. Cela implique une organisation en modules souple, aussi bien en ce qui concerne la distribution des modules entre les deux langues (qui se présentent toujours séparément) qu'en ce qui concerne leur place tout au long de ce cycle. La planification conjointe des deux langues et leur interrelation permettent une économie appréciable et donc rentabilisent les efforts, puisqu'en travaillant à partir de projets communs, les deux langues se renforcent mutuellement ; en effet, de nombreux savoir-faire linguistiques - ceux qui entrent en jeu dans les processus de production et de réception des textes oraux et écrits, ou dans les processus d'analyse et de réflexion sur la langue - ne sont le patrimoine exclusif d'aucune des deux. Au contraire, le travail parallèle et parfaitement programmé offre la possibilité de compléter et de comparer les connaissances acquises dans chacune des langues - langues qui, il faut le rappeler, possèdent une base commune ; chacun sait en effet qu'il s'agit de deux langues romanes qui ont subi une évolution historique parallèle et en interaction.

\section{Bachillerato et formation technico-professionnelle}

Dans le cycle du bachillerato (16-18 ans), tout comme pour l'enseignement secondaire obligatoire, l'ensemble langue catalane/langue castillane/littérature est envisagé comme une seule matière commune à tous les élèves.

17 Les contenus de la discipline, quant à eux, ont toujours un objectif très nettement orienté vers la communication, bien que l'on insiste dans cette étape sur « les contenus qui servent à stimuler la réflexion, la théorisation et la maitrise des structures linguistiques »; ils sont répartis en quatre blocs : phonétique et phonologie, lexique et sémantique, syntaxe, littérature. Les aspects communicatifs sont pris en compte dans le chapitre "méthodes»: compréhension de textes, analyse de textes, utilisation et transformation de textes, production et création de textes.

18 Pour ce qui concerne les principes théoriques sur lesquels s'appuient cette orientation et les aspects méthodologiques, les remarques faites pour le cycle ESO restent valables au niveau du bachillerato, bien qu'évidemment on insiste à ce niveau sur l'étude des aspects formels de la langue et de l'histoire littéraire. 
Quant à la formation technico-professionnelle, le champ disciplinaire "langue » ne figure que dans les cycles ou modules où l'utilisation de la langue est requise de façon spécifique et dans une optique professionnelle. L'enseignement de la langue présente alors toujours un caractère fonctionnel qui peut être identifié avec ce que l'on appelle l'enseignement de langue à des fins spécifiques.

\section{Défis présents et futurs}

Nous avons déjà signalé le degré d'autonomie et de souplesse de notre système éducatif en ce qui concerne l'enseignement des langues catalane et castillane. Nous avons aussi parlé de l'effort important de planification et de coordination que cela entraîne. Il semble de plus en plus évident et l'on a de plus en plus conscience que les résultats et les succès éducatifs sont étroitement liés à l'efficacité avec laquelle est réalisé le travail de coordination et de planification en question. Et c'est probablement à ce niveau que l'on ressent un peu d'inquiétude face à l'enseignement des langues dans notre pays.

21 Il faut d'abord signaler que, par rapport au système traditionnel d'autrefois, l'approche communicative dans l'enseignement des langues a fait un virage à 180 degrés, puisque ce sont non seulement les contenus et la méthodologie qui ont changé mais aussi, et de façon considérable, le rôle du professeur. Bien sûr, on ne saurait nier que dans de nombreux établissements, aujourd'hui, face à un ensemble de professeurs qui ont décidé d'opter délibérément pour les méthodes actuelles de l'enseignement communicatif, d'autres maintiennent les routines pédagogiques héritées des époques antérieures; c'est pourquoi les efforts de coordination et d'adaptation exigés par les temps nouveaux leur coûtent beaucoup. Il convient d'ajouter d'autre part que le travail de coordination et de planification pose quelques problèmes différents selon les zones et leur réalité sociolinguistique. Un des premiers problèmes rencontrés, par exemple, est celui du choix et de l'intégration des contenus dans la structure des « crédits ». Il ne faut pas oublier que bien que les situations et les niveaux de connaissances de départ soient différents, de nombreux élèves de l'ESO - et il va sans dire du bachillerato aspirent à aller à l'université ; or, pour y accéder, on exige au minimum qu'ils passent une épreuve commune lors de l'examen de selectividad.

Autre défi : faire en sorte que la responsabilité de l'enseignement de la langue soit partagée entre tous les professeurs. C'est dans cette optique qu'on a souvent entendu dire dans les établissements: "tout professeur est un professeur de langue». Malheureusement, malgré cela, cette affirmation est loin d'être valable partout. Le jour où cela sera réalité, peut-être observera-t-on chez les élèves un regain d'intérêt pour le champ disciplinaire de la langue.

Il existe un autre problème : il faut doter les établissements d'espaces adaptés et de moyens permettant d'étudier les langues en utilisant les nouvelles technologies (salles d'auto-apprentissage, salles informatisées, etc.). Certes, des efforts ont été faits dans ce sens, mais le rythme rapide des changements technologiques exige une attention et un renouvellement constants.

Il conviendrait enfin de signaler que, dans de nombreux établissements, il faudra améliorer le rapport entre la surface des salles de classe et le nombre d'élèves par classe, pour faciliter ainsi tous les types de travaux, individuels ou en groupe, dans ce 
qui devrait être un des principaux domaines de référence et d'interaction linguistique pour l'élève : la classe de langue.

\section{NOTES}

1. Dans cet article, nous nous référons exclusivement à la situation en Catalogne. Il faut pourtant noter que le catalan est aussi la langue d'autres régions autonomes espagnoles (Communauté valencienne et îles Baléares), et que d'autres régions en Espagne ont leur langue propre, différentes du castillan : la Galice et le Pays Basque. Même si, dans toutes ces Communautés, la législation linguistique et scolaire suit le même chemin, c'est en Catalogne que la langue minoritaire - le catalan - est la plus répandue, a la plus grande vitalité et où l'implantation généralisée d'un modèle scolaire bilingue est la plus achevée.

2. 2 J. Cummins, Linguistic Interdependance and the Educational Development of Bilingual Chidren, 1979.

3. 3 I. Villa, El català i el castellà en el sistema educatiu de Catalunya, 1995; J.-M. Serra, Inmersio lingüistica, rendiment acadèmic i classe social, 1997.

4. Curriculum du champ disciplinaire de langue, pour l'enseignement secondaire.

\section{RÉSUMÉS}

L'enseignement de deux langues officielles en Catalogne, le castillan et le catalan, requiert une planification adéquate. Les Projets linguistiques d'établissement et les Programmes d'immersion linguistique constituent les principaux instruments de la politique menée en ce sens par la Communauté autonome.

\section{INDEX}

Index géographique : Catalogne, Espagne

\section{AUTEURS}

\section{JOAN PERE}

Instituto de ciencias de la educacion, universidad de Barcelona, Espagne.

\section{MARÇAL FITÉ}

Instituto de ciencias de la educacion, universidad de Barcelona, Espagne 